\title{
Satisfacción del alumnado Erasmus con la orientación recibida en la USC
}

\author{
Satisfacción del alumnado Erasmus con la orientación recibida en la USC
}

\author{
Rebeca García-Murias, Luis Sobrado-Fernández, Elena Fernández-Rey \\ Universidade de Santiago de Compostela
}

\begin{abstract}
Resumen
Realizar una acción de movilidad requiere la puesta en práctica de competencias de diversa índole, entre ellas, las vinculadas con la toma de decisiones y la gestión de la información. La orientación, entendida como un proceso continuo a lo largo de la vida (Lifelong Guidance), se perfila en este contexto como uno de los factores necesarios para proporcionar a los estudiantes el apoyo y el asesoramiento que requieren en su proceso de toma de decisiones para afrontar con éxito las acciones de movilidad. Fomentar la intervención orientadora supone, además, avanzar hacia una mayor calidad de las instituciones de Educación Superior.

Palabras clave: movilidad, Erasmus, orientación
\end{abstract}

\begin{abstract}
Perform a mobility action requires the implementation of different competences, among which are those related to decision-making and information management. Orientation, understood as a continuous process throughout life (Lifelong Guidance), is profiled in this context as one of the necessary factors to provide students with the support and advice they require in the process of decision-making to address successfully the mobility actions. Encourage counseling intervention means also, move towards greater quality of Higher Education institutions.
\end{abstract}

Keywords: mobility, Erasmus, guidance

\section{Método}

La finalidad de esta comunicación es analizar el grado de satisfacción del alumnado que realizó una acción de movilidad Erasmus sobre la orientación recibida por parte de la universidad de origen, en este caso, de la Universidad de Santiago de Compostela (USC). Para ello, a partir de una aproximación teórica se desarrolló un estudio empírico con la entrevista como principal técnica de recogida de información. La muestra estuvo constituida por 23 estudiantes que ya habían realizado un intercambio a través de este programa. Sintéticamente se recogen los resultados obtenidos tras el análisis de contenido, haciendo hincapié, por un lado, en las razones que esgrimen los alumnos que se muestran satisfechos con esta orientación, y por otro, en los motivos de aquellos que indican su insatisfacción. Entre las principales razones de satisfacción manifestadas por el alumnado con respecto a la orientación recibida para su movilidad destacan: la información rápida y concisa, la aclaración de dudas e inquietudes y el asesoramiento y acompañamiento en todo el proceso. $\mathrm{Y}$, entre los principales motivos de insatisfacción se encuentran la escasa orientación recibida, el cambio de coordinador académico Erasmus y la falta de proximidad y escasa comunicación establecida. Por último, se extraen las principales conclusiones con el propósito de realizar una serie de propuestas de actuación y/o de mejora de la orientación universitaria para la promoción y difusión del programa de movilidad Erasmus.

\section{Resultados y discusión}

Para conocer la valoración que el alumnado otorga a la información y orientación recibidas, una vez llevada a cabo una acción de movilidad Erasmus, se toman como referencia los resultados derivados del análisis de las entrevistas.

Concretamente, en la primera cuestión se solicitó al alumnado que valorase su satisfacción con respecto a la orientación recibida desde su propia universidad a la hora de realizar la acción de movilidad (en una escala de cinco valores (1- nada satisfecho; 2-poco satisfecho; 3regular; 4-bastante satisfecho; 5- muy satisfecho).

Las apreciaciones obtenidas presentan un carácter positivo, como se pone de manifiesto con la media obtenida, alcanzando ésta un valor de 3.70 (próxima a "bastante" satisfecho).

En este sentido, cabe destacar que el 43.5\% ( $\mathrm{N}=10)$ de los sujetos entrevistados afirman haber recibido una orientación considerablemente satisfactoria desde la propia institución universitaria a la hora de realizar su estancia en otra universidad; mientras que sólo un $30.4 \%$ del alumnado ( $\mathrm{N}=7$ ) afirma no haber recibido una orientación lo suficientemente satisfactoria por parte de su /institución universitaria.

A continuación, se recogen los resultados obtenidos tras el análisis de contenido, haciendo hincapié, por un lado, en las razones que esgrimen los alumnos que se muestran satisfechos con esta orientación, y por otro, los motivos de aquellos que indican su insatisfacción.

Razones por las que el alumnado se encuentra satisfecho con la orientación recibida

En la figura 1 se sintetizan las principales razones de satisfacción del alumnado que ha sido entrevistado, con respecto a la orientación recibida de cara a la puesta en práctica de su movilidad.

Esta situación se refleja a través de diversos testimonios manifestados por los diferentes estudiantes:

"Desde el primer momento he recibido toda la información que he solicitado de forma rápida y no he tenido ningún problema en relación a la solicitud ni a la aceptación” (Sujeto 4).

"El coordinador-tutor de la USC que me correspondía siempre se mostró dispuesto a ayudarme 
en persona (...) estoy muy agradecida dado que siempre respondió rápidamente a mis dudas e inquietudes y trató de resolver los problemas con los que me encontré" (Sujeto 7).

"En mi caso me informaron correctamente de todo el papeleo que tenía que realizar, que era una buena elección y las convalidaciones no dieron ningún problema” (Sujeto10).

"Estoy satisfecho. Para mí se resume en rapidez, claridad y ayuda” (Sujeto 19).

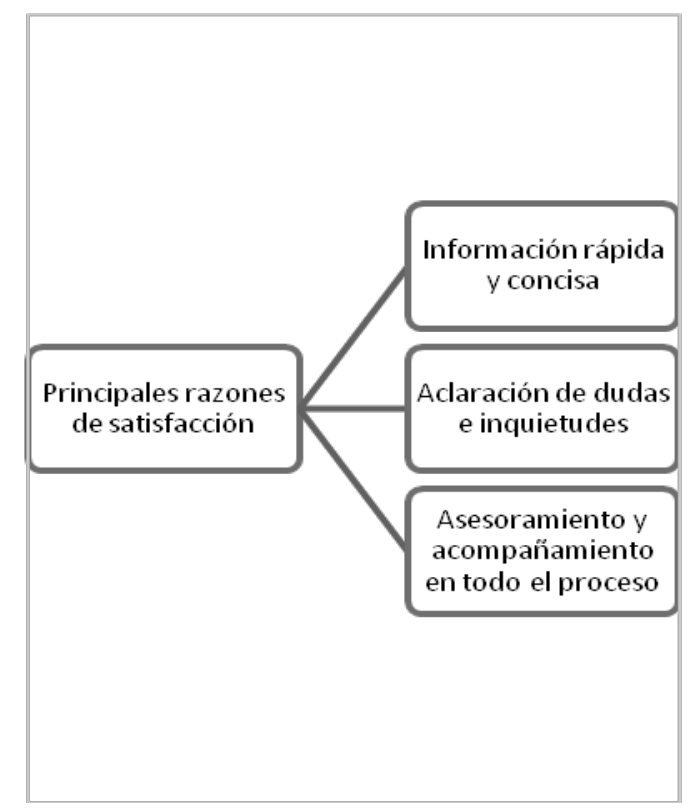

Figura 1: Orientación recibida de la universidad de origen (USC)

Razones por las que el alumnado se encuentra insatisfecho con la orientación recibida en su universidad de origen

En la figura 2 se aprecian los principales motivos de insatisfacción.

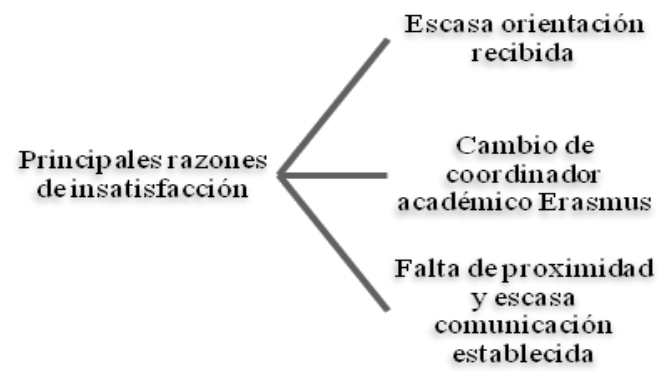

Figura 2: Orientación de la universidad de origen

Un elevado número de sujetos afirma sentirse poco satisfecho con las acciones de orientación propiciadas desde su propia institución universitaria (coordinadores Erasmus, Responsables Académicos de la Movilidad, Oficina y/o Servicio de Relaciones Exteriores, etc.) a la hora de realizar su estancia. Existe una relación de motivos por los cuales el alumnado de intercambio se siente desorientado al gestionar su movilidad. Éstos hacen referencia a cuestiones tales como los plazos y tiempos para llevar a cabo el proceso de solicitud de la movilidad, los requisitos y criterios de admisión y selección, la elaboración del acuerdo de estudios, las convalidaciones de las materias, la formación lingüística, las ayudas económicas o los aspectos logísticos.

Esta falta de orientación puede repercutir negativamente en su movilidad llegando, en ocasiones, a tomar la decisión de replantearse la posibilidad de llevar a cabo o no esta iniciativa o incluso durante su implementación a tener un sentimiento de no haber alcanzado las expectativas deseadas.

En este sentido, a continuación se incluyen algunos de los argumentos brindados por el alumnado al respecto:

"La sensación que me acompañó al tramitar el papeleo fue la indiferencia por parte de los coordinadores. Nunca tuve muy claro qué tenía que hacer o cómo. A la hora de realizar el acuerdo de estudios, por ejemplo, no recibí ningún tipo de asesoramiento. Se me indicó que mirara el programa de las asignaturas en la web de la universidad de acogida y eligiera en base a eso. Tuve bastantes dificultades para encontrar los contenidos referidos al ser usuario visitante sin clave de acceso..." (Sujeto 15).

"Creo que faltó profundizar en ciertos detalles de la movilidad, los métodos de estudio en la universidad de destino, así como una mejor orientación a la hora de la selección de materias, etc." (Sujeto 16).

"Penso que a ORE debía ofrecer cursos explicando no que consiste o Erasmus, mostrándoche o contexto cultural ao que te desprazas e as dificultades que podes atopar nos primeiros días. Non soamente dedicarse a firmar papeis senón desempeñar un rol máis amplo" (Sujeto 18).

En ocasiones, el estudiantado indica sentirse desorientado al no disponer del mismo coordinador académico durante su período en movilidad. Esta es una situación poco habitual, pero puede llegar a manifestarse repercutiendo, negativamente, en el proceso de seguimiento y asesoramiento hacia el alumnado en movilidad.

Como por ejemplo, el comentario al que alude el siguiente alumno:

"Las fechas presentan límites inflexibles (...) en mi caso hasta tuve cambio de coordinadores lo que conllevó a la no convalidación de tres de las cinco asignaturas cursadas en el primer semestre..." (Sujeto 13).

Otro de los aspectos ciertamente preocupantes es la falta de proximidad e interconexión y/o reciprocidad entre el alumnado interesado en llevar a cabo una iniciativa Erasmus y los coordinadores académicos de la movilidad. Esta situación es fruto, entre otros motivos, de la escasa o insuficiente formación de los gestores o responsables académicos comprometidos con las 
acciones de movilidad, la falta de disponibilidad del profesorado coordinador académico que llega a descuidar su labor orientadora con el estudiantado de intercambio y la ausencia de interés y compromiso por parte de éstos ante este tipo de iniciativas.

Muestra de ello queda patente en algunas de las respuestas emitidas por los jóvenes:

"Nos mandaron a un país con el que establecieron convenio por primera vez así que considero que la información ofrecida debería ser mayor. Mi acuerdo de estudios fue todo mal, puesto que todas las materias elegidas estaban en el idioma del país de acogida, gran problema ya que mi coordinador se intentaba poner en contacto con el de la USC y no era capaz (...). Por ello y más motivos considero que se debería ofrecer más información y orientación a los Erasmus y mantener más el contacto con ellos durante su estancia” (Sujeto 20).

Finalmente, ante esta primera cuestión, cabe señalar que el $26.1 \%(\mathrm{~N}=6)$ del alumnado restante presenta respuestas de carácter "neutro", esto es, ni manifiestan haber obtenido una orientación excesivamente positiva ni negativa por parte de su propia universidad de cara a la implementación de su programa de intercambio.

A modo de síntesis cabe señalar que, entre todos los alumnos que han sido entrevistados, un alto porcentaje afirma haber recibido una orientación lo suficientemente satisfactoria por parte de su institución universitaria. A pesar de esta situación, todavía son muchos los que manifiestan no estar lo adecuadamente orientados en este proceso, reclamando en cierto modo, un mayor y mejor asesoramiento por parte de su universidad para que su movilidad se desarrolle con éxito.

\section{Conclusiones}

Tal y como se ha recogido en este trabajo, nuestro interés se centró en conocer el grado de satisfacción de los estudiantes que ya habían disfrutado de una experiencia de movilidad Erasmus en relación a la orientación recibida desde la universidad de origen, en este caso, la USC.

Las apreciaciones del alumnado en movilidad presentan, en general, un carácter positivo. No obstante, se centra la atención en aquellos estudiantes que manifiestan mayores necesidades al respecto $\mathrm{y}$, por consiguiente, se precisa optimizar esta situación.

En este sentido y tal y como se ha apuntado previamente, existe un importante porcentaje de alumnado que afirma no haber recibido una orientación lo suficientemente satisfactoria por parte de la universidad de origen para la realización de la movilidad Erasmus. Entre las principales razones por las que los estudiantes se muestran insatisfechos destacan la escasa orientación recibida, el cambio de coordinador académico Erasmus, la falta de proximidad y la insuficiente comunicación establecida.

En definitiva, los resultados obtenidos permiten sostener la importancia de establecer un mayor compromiso institucional a través de políticas de la propia universidad, facilitando los recursos materiales y humanos que lo hagan posible, optimizando la gestión del programa Erasmus a través de, por ejemplo, la mejora de la calidad de las políticas de promoción y difusión de la información y orientación acerca de las acciones de movilidad y programas de intercambio - no sólo entre su alumnado sino también en los restantes grupos que conforman la comunidad universitariaofreciendo una dimensión europea de sus enseñanzas de cara a alcanzar una verdadera convergencia europea

El diseño de estrategias orientadoras destinadas a la movilidad desde la institución universitaria resulta un aspecto clave para la promoción y difusión de las políticas de información y orientación que apoyen las acciones de movilidad académico-profesionales y asesoren al alumnado que decide llevar a cabo una estancia en Europa (García Murias, 2012; 2013). Entre otras, la creación de servicios de orientación en el ámbito universitario se perfila como una de las estrategias más idóneas para ofrecer respuesta a las necesidades y demandas del alumnado en movilidad (Sobrado \& Barreira, 2012), pudiendo establecer acciones de seguimiento y orientación, aspecto de sumo interés y cuya finalidad se centra en conocer las implicaciones que, en el ámbito académico, formativo, profesional, social, cultural, etc., conllevó la puesta en práctica de su movilidad. La tutorización de la movilidad es un tema pendiente para las universidades que, de acuerdo con Senent (2015), puede situarse entre dos polos: la movilidad autónoma del alumnado y la inversión por parte de las instituciones públicas en la formación de docentes (y otros agentes) que fomenten y participen en procesos de movilidad.

Otras actuaciones orientadoras podrían ir encaminadas a promover las tutorías entre iguales, crear un servicio de apoyo y asesoramiento a los coordinadores académicos y demás personal responsable de gestionar la movilidad para mejorar sus competencias y su labor profesional o promover un Plan de Acción Tutorial en la Universidad, entre otras.

Atendiendo a los diversos testimonios del alumnado entrevistado podemos concluir igualmente la inmediata necesidad de potenciar la figura del coordinador académico del programa de movilidad Erasmus a partir de la definición de su perfil y de unas determinadas competencias. Esto es, se precisa dotar a los coordinadores académicos de la movilidad de una formación específica (tanto a nivel pedagógico como de gestión) vinculada con los procesos de asesoramiento y orientación hacia el alumnado en movilidad. Esto es, a partir del análisis de necesidades diseñar acciones formativas organizadas, con el objeto de ofrecer una mejor orientación al alumnado en movilidad.

Finalmente, consideramos que potenciar el uso de las Tecnologías de la Información y de la Comunicación (TIC) en las acciones de movilidad facilitaría acceder a la información disponible sobre estas iniciativas europeas, así como entablar un mayor intercambio de conocimientos y experiencias entre los profesionales y el alumnado en movilidad. 


\section{Referencias}

García Murias, R. (2012). La Orientación Educativa a lo largo de la vida. En L. M. Sobrado, E. Fernández Rey \& M. L. Rodicio (Coords.) Orientación Educativa: Nuevas perspectivas (pp. 21-41). Madrid: Biblioteca Nueva.

García Murias, R. (2013). Exploración de las necesidades de información del alumnado del programa Erasmus y propuestas de actuación orientadora. Tesis Doctoral, Universidade de Santiago de Compostela, Santiago de Compostela.
Senent, J. (2015). Movilidad de estudiantes: Microanálisis del programa Erasmus (2009-2014). Estudio de caso. Bordón, 67 (1), 117-134.

Sobrado, L. M., \& Barreira, A. (2012). Servicios de Orientación educativa: Organización y funcionamiento. En L. M. Sobrado, E. Fernández Rey \& M. L. Rodicio (Coord.), Orientación Educativa, Nuevas Perspectivas (pp.187-214). Madrid: Biblioteca Nueva. 